\title{
Early Visual Cortex as a Multiscale Cognitive Blackboard
}

\author{
Pieter R. Roelfsema ${ }^{1,2,3}$ and Floris P. de Lange ${ }^{4}$ \\ ${ }^{1}$ Netherlands Institute for Neuroscience, 1105 BA Amsterdam, The Netherlands; \\ email: p.roelfsema@nin.knaw.nl \\ ${ }^{2}$ Department of Integrative Neurophysiology, VU University Amsterdam, \\ 1081 HV Amsterdam, The Netherlands \\ ${ }^{3}$ Psychiatry Department, Academic Medical Center, 1105 AZ Amsterdam, The Netherlands \\ ${ }^{4}$ Donders Institute for Brain, Cognition and Behavior, Radboud University, \\ 6525 EN Nijmegen, The Netherlands
}

Annu. Rev. Vis. Sci. 2016. 2:131-51

First published online as a Review in Advance on July 18, 2016

The Annual Review of Vision Science is online at vision.annualreviews.org

This article's doi:

10.1146/annurev-vision-111815-114443

Copyright (c) 2016 by Annual Reviews. All rights reserved

\section{Keywords}

visual cortex, cortical layers, visual routines, working memory, decision-making

\section{Abstract}

Neurons in early visual cortical areas not only represent incoming visual information but are also engaged by higher level cognitive processes, including attention, working memory, imagery, and decision-making. Are these cognitive effects an epiphenomenon or are they functionally relevant for these mental operations? We review evidence supporting the hypothesis that the modulation of activity in early visual areas has a causal role in cognition. The modulatory influences allow the early visual cortex to act as a multiscale cognitive blackboard for read and write operations by higher visual areas, which can thereby efficiently exchange information. This blackboard architecture explains how the activity of neurons in the early visual cortex contributes to scene segmentation and working memory, and relates to the subject's inferences about the visual world. The architecture also has distinct advantages for the processing of visual routines that rely on a number of sequentially executed processing steps. 


\section{INTRODUCTION}

Humans and nonhuman primates are visual creatures: Vision is our dominant sense, both in terms of perception (e.g., Rock \& Victor 1964) and in terms of the amount of neural circuitry dedicated to it (Kaas 2008). For example, in the macaque monkey, the presence of visually responsive neurons and major inputs from known visual areas indicates that more than 30 separate neocortical areas are implicated in visual processing, so more than $50 \%$ of the cerebral cortex contributes to visual processing (Felleman \& Van Essen 1991). Although all these cortical areas are undoubtedly relevant for the processing of visual information, recent findings have suggested that many of them may also play an important part in cognitive processes that are not strictly visual, such as working memory or decision-making, thus blurring the boundaries between perception and cognition (Mesulam 2008).

The early visual cortex receives information from higher cortical areas through numerous feedback projections, at least as many as the feedforward projections from lower to higher areas (Markov et al. 2011). For example, there are many feedback projections to the primary visual cortex, originating from areas within the visual system [e.g., V2-V4, V5 (or MT)], from other sensory cortices (e.g., the primary auditory cortex), and also from nonsensory areas in the parietal and frontal cortices (Markov et al. 2011, Muckli \& Petro 2013). Thus, the early visual cortex interacts with several other cortical systems, but the role of feedback connections is only partially understood.

We review and extend a theoretical framework in which feedback connections enable the early visual cortical areas to act as an active blackboard (Bullier 2001, Cavanagh 2011, Kosslyn et al. 2001, Mumford 1991, Van der Velde \& de Kamps 2006) where task-relevant features are highlighted with enhanced neuronal activity. The great advantage of such a blackboard is that early visual areas encode low-level, general-purpose features. Higher areas can write to the blackboard by activating or enhancing the representation of those features that are currently of importance and by suppressing features that are not. Other higher areas can then read out these task-relevant features for further processing. This blackboard function circumvents the need for direct interactions between higher areas that process unrelated features, which may be difficult to implement. We review evidence in favor of such a retinotopic cognitive blackboard, drawing on previous results from tasks involving scene segmentation, selective attention, working memory (Harrison \& Tong 2009), mental imagery (Albers et al. 2013, Pearson et al. 2015), decision-making (Lorteije et al. 2015, Nienborg \& Cumming 2009), and visual routines (Moro et al. 2010). We examine how feedback signals from higher areas to the early visual cortex can be interpreted in terms of write operations to the blackboard, which can then be read by other cortical areas and subcortical structures in later processing steps, thereby supporting the exchange of intermediate computational results.

\section{SCENE SEGMENTATION}

When we see a new image, cortical processing goes through different phases. The first phase is dominated by feedforward connections that propagate information from the lateral geniculate nucleus (LGN) of the thalamus to the primary visual cortex (area V1) and then onward to higher visual areas. During this feedforward sweep, low-level areas extract basic visual features, such as orientation, motion, and color, and higher visual areas extract more complex, semantic features, such as the presence of faces, animals, and other object categories (Hung et al. 2005, Thorpe et al. 1996; reviewed in Roelfsema 2006). During the next, recurrent phase, horizontal and feedback connections come into play. Feedback connections propagate activity from higher areas back to lower areas, and horizontal connections enable interactions within the areas, for example between cells with different receptive field locations. 
These different connection types provide distinct contributions to visual perception. Take, for example, the texture-segregation task in Figure 1 $\boldsymbol{a}$, where we perceive a figure composed of line elements that differ in their orientation from the background. Psychophysical (Mumford et al. 1987, Wolfson \& Landy 1998), neurocomputational (Bhatt et al. 2007), and neurophysiological (Lamme 1995, Poort et al. 2012, Zipser et al. 1996) studies have demonstrated that figure-ground segregation relies on a number of processing steps. First, the feedforward sweep activates orientation-selective neurons in low-level areas with small receptive fields and in higher visual areas that represent the image at a coarser resolution (Figure $\mathbf{1 b}$ ). Then, the image is represented at multiple resolutions in an image-processing pyramid (Burt \& Adelson 1983, Hochstein \& Ahissar 2002). The next step is boundary detection. This process is sensitive to changes in orientation at locations where the figure and background abut. The network can detect boundaries if there are inhibitory connections between neurons that are tuned to the same orientation and whose receptive fields are adjacent to each other (Figure $\mathbf{1} b$ ). Inhibition is strong in regions with a homogeneous orientation, such as the background and the center of the figure, but weaker at the boundaries (Itti \& Koch 2001, Roelfsema et al. 2002). As a result, neuronal activity is highest at the boundaries between figure and background (Figure 1 $\boldsymbol{a}, \boldsymbol{b}$ ). In this phase, the representation of the center of the figure is still suppressed in lower visual areas, including V1. However, neurons in higher areas that respond to the figure center increase their activity. They have larger receptive fields, and this has two consequences. First, the neurons do not resolve local figure-ground boundaries, which are, therefore, blurred. Second, neurons whose receptive fields cover the figure receive very little inhibition from neighbors that represent pure background, causing an enhanced activity level that psychologists call pop-out (Itti \& Koch 2001, Lee et al. 2002, Poort et al. 2012).

The ensuing process is called region filling, and it relies on feedback connections from neurons in higher visual areas that have increased activity due to pop-out (Figure 1 $b$ ). The feedback inputs amplify the activity of neurons in lower areas that code for the figure's line elements at increasingly precise spatial scales (Figure 1 b). Next, the entire figural region is labeled with enhanced neuronal activity at multiple scales. This region-filling process appears to be important for behavior because the distribution of enhanced V1 activity across the figure predicts the landing position of the eye if an eye movement is made toward the figure (Poort et al. 2012). If the response enhancement is stronger on the right side of the figure, the landing position is biased to the right, and if the response modulation is stronger on the left, the gaze tends to land on that side. Furthermore, if the task demands that the animal directs attention away from the figure, region filling is weaker, as if the representations of unattended objects are left in a more primordial state (Figure 1a). In the blackboard metaphor: Higher visual areas write to the early visual cortex to highlight the features of the figure. The enhanced activity is the chalk on the blackboard. Eye movement processing centers, such as the superior colliculus and the frontal eye fields, read the pattern of enhanced activity to determine the target for an eye movement, enabling the eye to land on the center of gravity of the region that is labeled with enhanced activity.

Boundary detection and region filling require different computations (Roelfsema et al. 2002), and the visual brain apparently implements these two processes through different connection types and at different times. Furthermore, feedforward drive from the LGN strongly depends on AMPA receptors, whereas the feedback influence for region filling depends more strongly on NMDA receptors (Self et al. 2012). Finally, the different phases of texture segregation involve different layers of the cortex (Self et al. 2013). The activity from the LGN arrives in V1 in layers 4 and 6 , and neurons in these layers then quickly propagate activity to the other cortical layers (Figure 1c). Next, the boundaries are detected in the superficial layers of the cortex, which amplify the representation of the boundaries. This is followed by the region-filling process that involves feedback from higher visual areas to layers 1 and 5, which are known to be the targets of feedback 
a

Stimulus Spontaneous activity Visual response Boundary detection Region filling

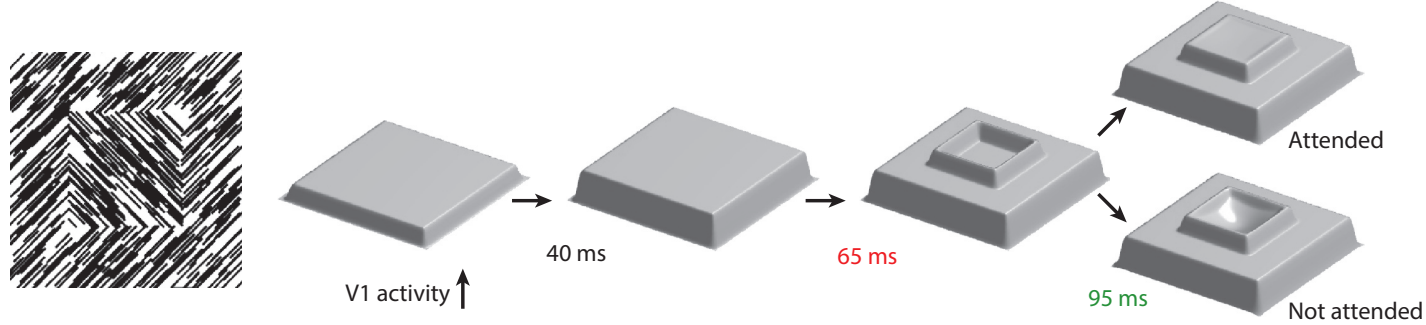

b

Visual response and

boundary detection Region filling

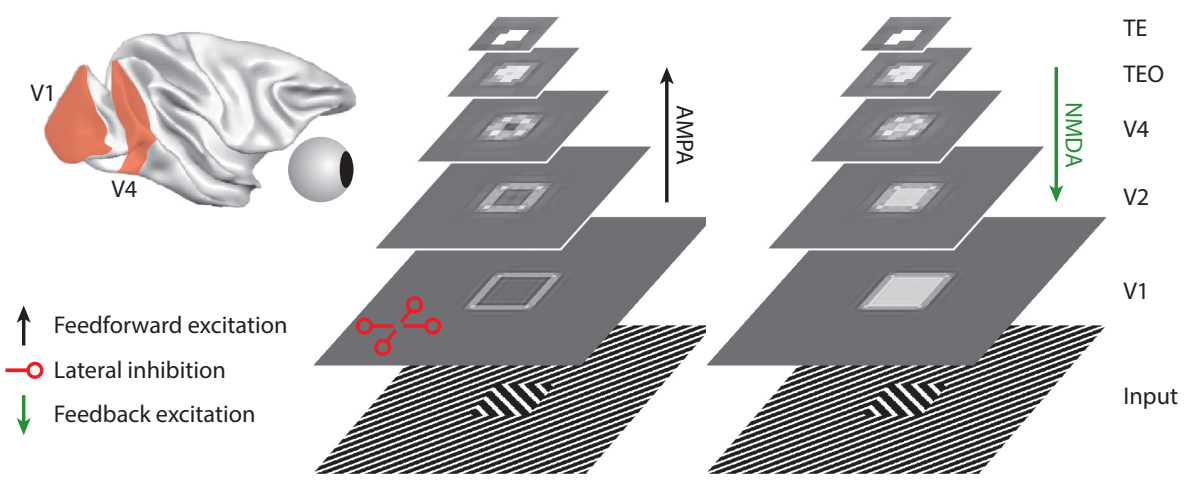

C

Visual response

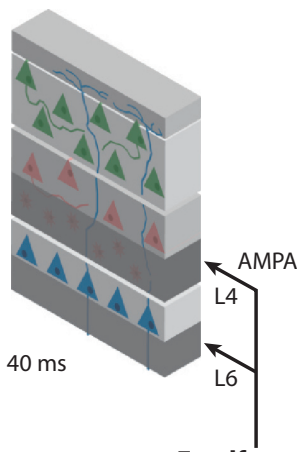

Feedforward

(lateral geniculate nucleus)
Boundary detection

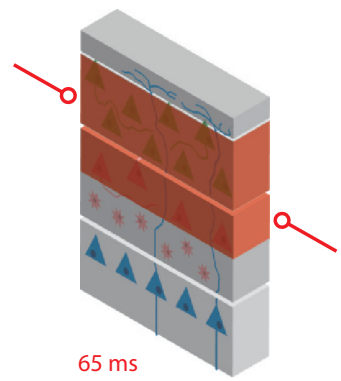

Region filling

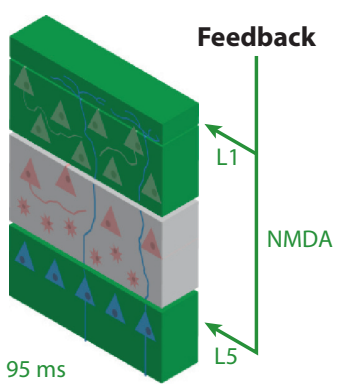


connections (Rockland \& Virga 1989). Region filling enhances the activity elicited by the figure in all cortical layers, but most strongly in the superficial and deep layers (Self et al. 2013).

It is an open question whether a similar succession of processes occurs for images other than these simplified texture stimuli. Recent progress in deep learning has been made in the recognition of semantic categories in photographs by using neural networks consisting of many layers (LeCun et al. 2015). The representations formed by these deep networks resemble those found along the visual cortical processing hierarchy (Güçlü \& van Gerven 2015, Yamins et al. 2014), and computer vision studies have started to use semantic labels for image segmentation. These algorithms can register which pixels belong to the various objects in a scene, using a process that resembles the influence of corticocortical feedback (Long et al. 2014). We expect that new insights into the processes of scene segmentation in the visual cortex will be obtained by comparing neurophysiological findings to the organization of these deep networks.

\section{OBJECT-BASED ATTENTIONAL SELECTION}

Some perceptual grouping tasks rely on a more prolonged process of recurrent interaction (Chen et al. 2014). One example is the curve-tracing task illustrated in Figure $2 \boldsymbol{a}$. If you want to switch on the hair dryer, would you pick up the white or black plug? To identify the correct plug, participants trace the relevant cable by gradually spreading object-based attention over its representation (Houtkamp et al. 2003). Reaction time increases with the length of the curve, indicating a serial process (Jolicoeur et al. 1991). Similarly, in the visual cortex of monkeys, curve tracing is associated with the gradual spread of enhanced neuronal activity over the representation of the curve (Pooresmaeili \& Roelfsema 2014, Roelfsema et al. 1998). Neurons with receptive fields located near the start of the curve exhibit an earlier enhancement of their response than neurons with receptive fields farther along the curve, and eventually the entire curve is labeled with enhanced neuronal activity (Figure $2 \boldsymbol{b}$ ). Importantly, the propagation speed of this labeling process depends on the distance between the curves. If the traced curve is near a distracting curve, the tracing speed is lower, but the speed is higher when the curves are farther apart (Jolicoeur et al. 1991, Pooresmaeili \& Roelfsema 2014). As a result, the processing time in Figure $2 \boldsymbol{a}$ is independent of the viewing distance (Jolicoeur \& Ingleton 1991). If you look at the image from a distance of, say, $1 \mathrm{~m}$, you have to trace a short curve (measured in degrees of visual angle), but it is close to the distractor. If you view it from $25 \mathrm{~cm}$, its length in degrees increases by a factor of 4 , but the distance between the curves increases by the same factor, so that the total tracing time remains roughly the same.

We can explain this scale-invariant tracing speed if tracing relies on the blackboard's multiple scales. The tracing process involves neurons with different receptive field sizes at different levels of the visual cortical hierarchy. Figure $2 c$ illustrates this idea. Initially, the curves are far apart,

\section{Figure 1}

Neuronal computations for texture segregation. (a) Stimulus with a square figure composed of line elements with one orientation superimposed on a background with a different orientation (left). The other subpanels illustrate the successive processing phases in V1. First, features are registered, and then V1 activity increases at the boundaries. Finally, region filling occurs, so that the V1 representation of image elements in the figure's interior is also enhanced. Region filling is incomplete for nonattended figures (Poort et al. 2012). (b) Feedforward connections use AMPA receptors to propagate activity to higher areas with larger receptive fields (black arrow). Boundary detection (and pop-out) occurs at each level because neurons that are tuned to the same orientation inhibit one another (red connections). Feedback connections depend on NMDA receptors, and they enhance activity at the figure's center for region filling (green arrow) (Self et al. 2012). (c) Features are first registered in layers 4 and 6 of area V1 (gray). Boundary detection (red) occurs slightly later in the superficial layers, and region filling ( green) occurs due to feedback to the superficial layers and layer 5 , causing an enhancement of firing rates that is most prominent in the superficial and deep layers (Self et al. 2013). 
a

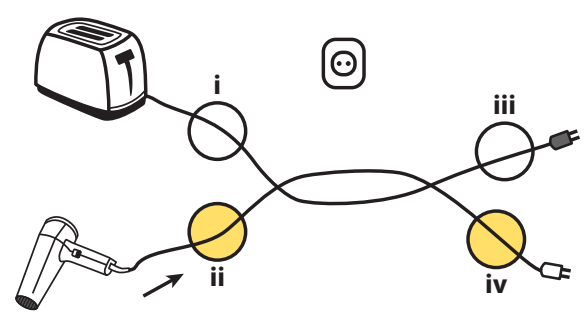

b

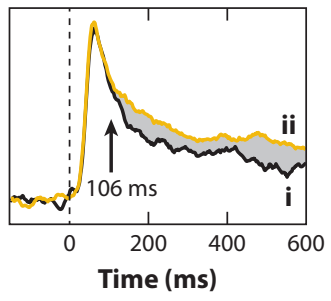

Area V1

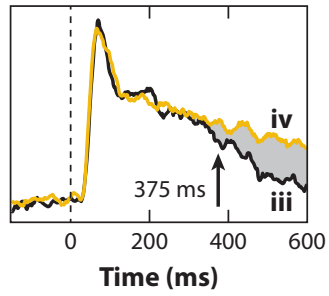

C
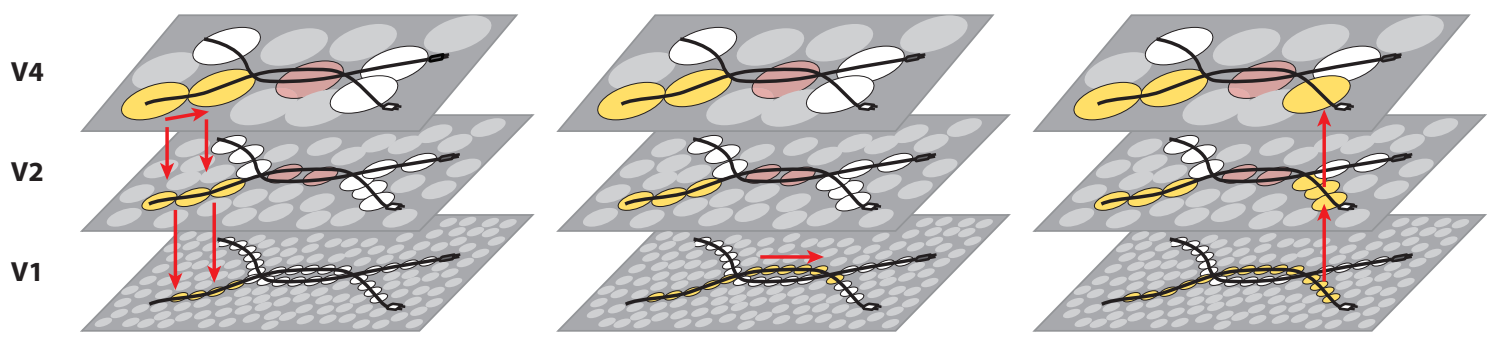

Time $\longrightarrow$

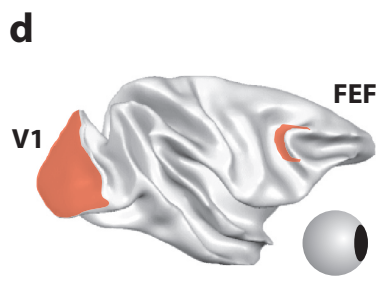

V1

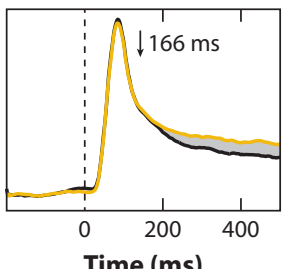

FEF

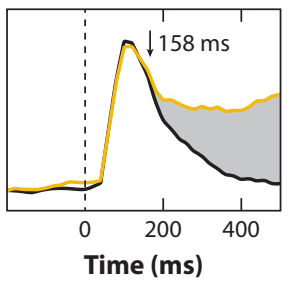

Figure 2

Neuronal mechanisms for object-based attention at multiple spatial scales. (a) A serial process is invoked in a curve-tracing task. Here, the task is to determine which plug is connected to the hair dryer. (b) V1 neurons enhance their response if the RF falls on the target curve (left, yellow response; see the RF labeled ii in panel $a$ ) compared with when it falls on the distractor (black response; i in panel $a$ ). The response enhancement occurs at a later time point for RFs farther along the curve (right; RFs labeled iii, iv in panel $a$ ). (c) Contour grouping proceeds at a high speed if curves are far apart. Presumably, it relies on the propagation of enhanced activity among neurons in higher areas with large RFs. Yellow circles represent RFs that have been reached by the propagating wave of enhanced activity. These neurons also send feedback to neurons in lower areas, enhancing the activity elicited by the relevant curve (red downward arrows). If curves are close to each other, multiple curves fall within one RF in the higher areas, so neurons in lower areas with smaller RFs take over because propagation in the higher areas is blocked (red circles). Propagation in the lower areas is slower because it relies on neurons with smaller RFs. If the response modulation reaches a region of the image where the distance between curves is larger, the higher areas can resume the propagation process, and its speed increases (Pooresmaeili et al. 2014). (d) If curves are far apart, the spread of object-based attention has similar timing in V1 and the FEFs (Pooresmaeili \& Roelfsema 2014). Abbreviations: FEF, frontal eye field; $\mathrm{RF}$, receptive field. 
and tracing proceeds at high speed in the higher visual areas where the receptive fields are large. Neurons in lower areas receive feedback from higher areas (Figure $2 c$ ) so that the propagation is also fast. When the curves are near to each other, however, the larger receptive fields cannot resolve the individual curves and propagation in higher areas is blocked. According to this model, the lower areas have to take over, but at a slower speed due to their smaller receptive fields. Farther along the curve, the distance between the curves increases again, so the higher areas can resume their contribution to the tracing process. Measurements of the propagation delays in monkey V1 during the curve-tracing task have provided strong evidence in favor of such a multiscale blackboard model, revealing a horizontal propagation of approximately $50 \mathrm{~ms}$ per receptive field at the level where the fastest progress is made (Pooresmaeili \& Roelfsema 2014).

A recent study made simultaneous recordings in $\mathrm{V} 1$ and the frontal eye fields (FEFs), a frontal area involved in eye movement planning, during curve tracing (Pooresmaeili et al. 2014). Remarkably, the latency of attentional selection (i.e., the labeling process) in the visual and frontal cortices was similar (Figure $2 \boldsymbol{d}$ ). Yet in V1, the amplitude of the attentional signal was smaller than in the FEF, and it saturated early during the trials. The selection signal in the FEF kept increasing until the behavioral response (an eye movement), as if the neurons in the FEF were integrating attentional signals in the visual cortex (reading from the blackboard). The delay between the V1 and FEF neurons that represented the same curve was remarkably short, much shorter than that between V1 neurons representing different segments of an elongated curve (Figure $2 \boldsymbol{b}$ ). However, this near-simultaneity broke down if the monkey made an error. In error trials, enhanced activity for the wrong curve first appeared in the FEF, which then imposed the erroneous selection signal onto the visual cortex, where it was expressed more than 100 ms later (Pooresmaeili et al. 2014, Roelfsema \& Spekreijse 2001). The fact that selection signals in the FEF influence attentional signals in the visual cortex also follows from studies that used electrical microstimulation to perturb activity. If the activity of the FEF neurons with a receptive field at a particular location is increased with microstimulation, the activity of neurons in the visual cortex that represent the same location also increases (Armstrong et al. 2006, Ekstrom et al. 2008, Moore \& Armstrong 2003). Thus, the reciprocal interactions between the visual cortex and higher areas enable the coselection of the same stimulus across different brain regions, which is essential for perceptual organization (Duncan et al. 1997).

The slow, serial processing that characterizes curve tracing generalizes to other perceptual grouping tasks. It is relatively easy to construct stimuli for which comparable delays occur during grouping by proximity, common fate, and similarity (Houtkamp \& Roelfsema 2010), and these grouping cues cause a comparable spread of attentional selection signals in the visual cortex (Gilad et al.2013, Li et al.2006, Wannig et al. 2011). In all these cases, perceptual grouping is accompanied by the labeling of the to-be-grouped image elements in the visual cortex with enhanced neuronal activity (the precise relationship between object-based attention and perceptual grouping has been discussed in Roelfsema \& Houtkamp 2011). Again, the lower visual areas appear to provide a blackboard to which the higher areas write the outcome of their computations, establishing groupings by labeling neurons with enhanced activity. Neurons in the lower areas could actively participate in this labeling process through horizontal connections, and higher areas could read from this blackboard whenever subsequent mental processing steps or overt behavior depended on the established groupings, for example for planning an eye movement (Pooresmaeili et al. 2014, Poort et al. 2012) or arm movement toward the correct plug in Figure $2 a$. So far, the evidence for a causal role of these response modulations in perceptual grouping has remained indirect. Future studies might test whether interfering with the modulation of activity in the early visual cortex impairs perceptual grouping and object-based attention. 


\section{MAINTAINING AND MANIPULATING VISUAL INFORMATION}

The sections above illustrate how the early visual cortex can assist in complex perceptual operations. However, many cognitive processes do not act upon information that is immediately available to the eyes, but rather on information that is kept in working memory. Do early visual areas also have a role in working memory and can they contribute to the maintenance and manipulation of information that is not directly visible? Traditionally, these mnemonic functions have been ascribed to prefrontal neurons that show sustained, elevated neural firing during the maintenance of visual information (Goldman-Rakic 1995), but the evidence for working memory in sensory areas is more equivocal (Pasternak \& Greenlee 2005). Several studies have shown that activity in the early visual cortex of awake-behaving monkeys during the maintenance of sensory material is at baseline levels (Lee et al. 2005), or weak and transient (Ferrera et al. 1994, Zaksas \& Pasternak 2006), unless the to-be-remembered stimulus remains on the screen (Supèr et al. 2001). A recent study has observed sustained, working-memory-related spiking activity in the MST region but not the earlier sensory MT region, suggesting a sharp boundary between early visual areas, which are involved in perception, and late visual areas, which are involved in mnemonic processes (Mendoza-Halliday et al. 2014).

Contrary to this notion, however, recent data from the Roelfsema lab have indicated elevated spiking activity in V1 during the maintenance of visual material in memory (van Kerkoerle et al. 2014). In support of this view, neuroimaging studies in humans have uncovered orientationselective activity patterns in the early visual cortex (V1-V4) during the maintenance of oriented stimuli in working memory (Albers et al. 2013, Harrison \& Tong 2009, Serences et al. 2009). Note, however, that this finding might arise from the nature of the measurements: BOLD (blood oxygen level-dependent) signals represent a mass action signal that is sensitive to variations in the firing rate of neurons but also to synaptic activity that does not translate into spiking (Logothetis \& Wandell 2004), and that is manifested only in the local field potentials (Mendoza-Halliday et al. 2014). Although more research is needed, it is conceivable that the involvement of brain regions in maintaining visual information depends on the exact stimulus properties and task demands, rather than there being a strict dividing line between sensory and memory-related regions. Mnemonic activity in early visual areas might be necessary in tasks that require memory for low-level features and a high spatial resolution.

Humans and nonhuman primates (Rainer et al. 1999) not only are able to maintain information over time, but also can mentally manipulate internally stored visual information. For example, when determining whether the letters depicted in Figure $\mathbf{3} \boldsymbol{a}$ are in normal or mirror image form, participants typically report mentally rotating the letters into their upright form and inspecting the resulting mental image (Kosslyn 1996). In line with this suggestion, the time required for these judgments monotonically increases with the amount of mental rotation required (Shepard \& Cooper 1982, Shepard \& Metzler 1971).

Are early visual cortical areas involved in the mental manipulation of visual content? Recent neuroimaging studies in humans have indicated that this is indeed the case (Albers et al. 2013, Stokes et al. 2009). For example, Albers et al. (2013) compared the cortical representation in the early visual cortex during working memory and mental imagery to that elicited by bottomup stimulation. Participants saw images of oriented gratings, which they had to memorize or rotate mentally (Figure $3 \boldsymbol{b}$ ). Overall levels of BOLD activity in the visual system were increased after presentation of the stimuli, but dropped in the intervening maintenance or manipulation interval (Harrison \& Tong 2009). Importantly, the pattern of BOLD activity across voxels in the early visual cortex during this interval reliably reflected the stimulus in working memory (Figure $3 c$ ), or, in the case of mental rotation, what the stimulus looked like after mental rotation 
a

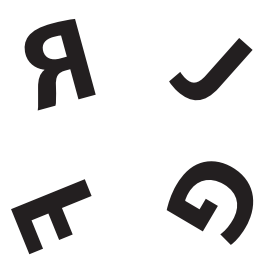

b

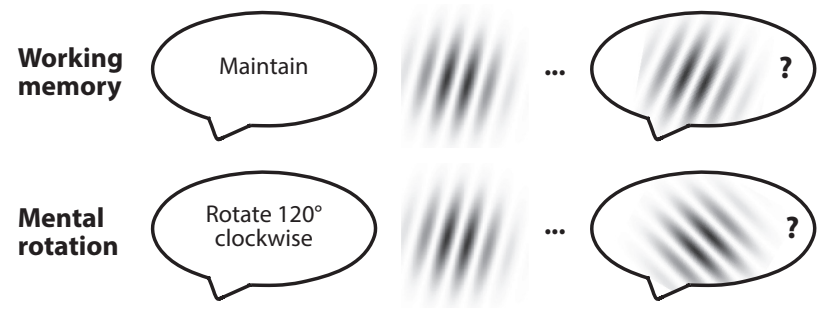

C

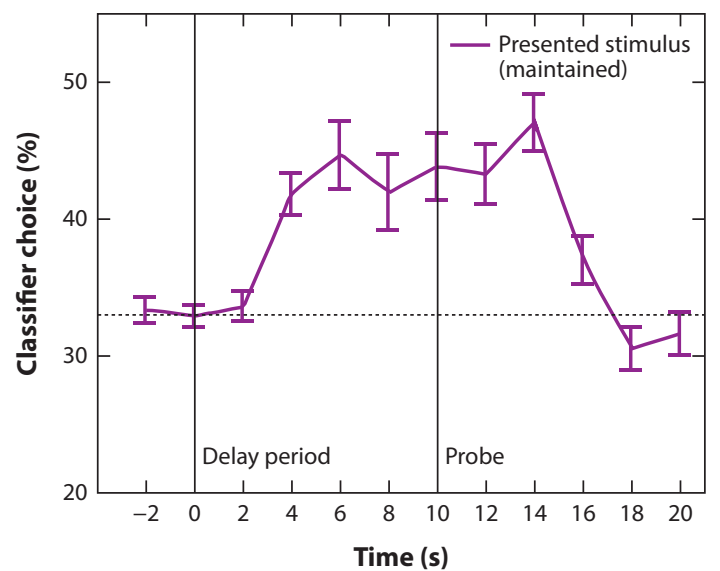

d

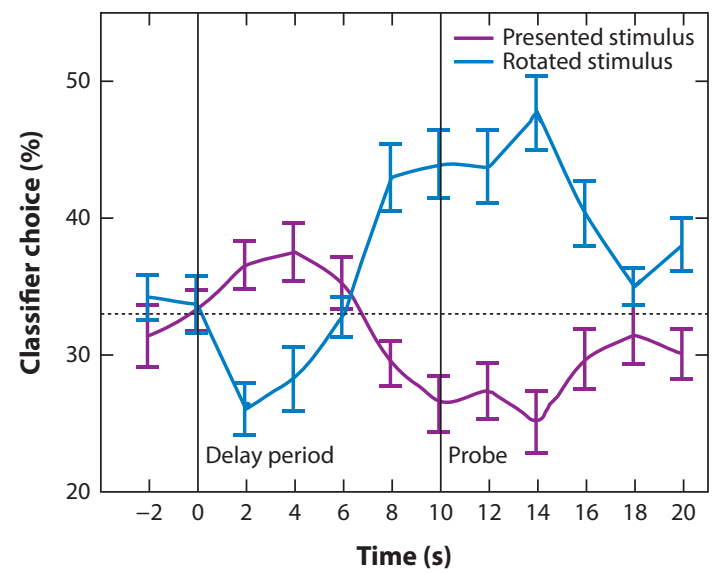

Figure 3

Mental manipulation of images. (a) Example of a mental rotation test. Are these letters in normal or mirror image form? Participants respond more quickly to the top row $(\mathrm{R}$ is the mirrored letter, and $\mathrm{J}$ the canonical letter) than the bottom row ( $\mathrm{F}$ is the mirrored letter, and $\mathrm{G}$ the canonical letter) due to the smaller amount of mental rotation necessary to turn the letters to their upright position. (b) Task setup of Albers et al. (2013). Participants had to either memorize a visually presented grating for $10 \mathrm{~s}$ (working memory) or mentally rotate the grating and imagine the rotated stimulus. After the memory delay, the participants had to compare the orientation that they had in mind to the orientation of a probe. BOLD (blood oxygen level-dependent) activity patterns in the visual cortex were recorded during task performance using functional magnetic resonance imaging. (c) During working memory, BOLD activity patterns in the visual cortex resembled the bottom-up activity patterns of the stimulus that was maintained in working memory. (d) During mental rotation, activity patterns in the visual cortex initially resembled the bottom-up activity patterns of the presented stimulus, but subsequently resembled the mentally rotated stimulus.

(Figure $3 \boldsymbol{d}$ ). Moreover, these BOLD patterns were similar to those elicited during bottom-up visual stimulation, implying a common internal representation for perception and imagery.

Although mental imagery is difficult to assess in nonhuman primates, several studies have suggested that monkeys do indeed also form mental images. For example, when arrows become associated with certain motion directions via learning, presentation of an arrow can elicit directionselective activity of MT neurons tuned to the respective motion direction (Albright 2012, Schlack \& Albright 2007). Related findings have been obtained in the inferotemporal (Sakai \& Miyashita 1991) and frontal cortices (Rainer et al. 1999). For example, Rainer et al. (1999) trained monkeys to associate a sample stimulus with a test stimulus. After training, the presentation of the sample 
stimulus gradually recruited neurons representing the test stimulus during a memory epoch when monkeys anticipated making a judgment based on this test stimulus.

Does the modulation of activity in the early visual cortex during working memory and imagery have a causal influence or is it an epiphenomenon caused by the presence of feedback connections from higher cortical areas? The use of transcranial magnetic stimulation (TMS) that disrupted activity in the early visual cortex during imagery (Kosslyn et al. 1999) and working memory tasks (van de Ven et al. 2012) has suggested that activity in the early visual cortex is indeed read out and influences task performance, although this is still an active topic of ongoing research.

These results, taken together, provide support for our hypothesis that early visual areas can serve as a blackboard for the information generated by bottom-up perception and are involved not only in the modulation of this information by attention but also during higher level cognitive processes, including working memory and imagery (Bullier 2001, Harrison \& Tong 2009, Roelfsema 2005).

\section{PERCEPTUAL DECISION-MAKING}

Perceptual decision-making refers to the deliberate and effortful process of collecting sensory evidence over time or space, resulting in the commitment to a categorical proposition (Gold \& Shadlen 2007, Schall 2001). Decision-making is intimately related to working memory because it requires integrating previous sensory evidence in memory with new evidence that comes in. Much progress has been made in terms of understanding how we accrue and weigh sensory evidence and how and when a decision is made. For example, when a link is imposed between a perceptual decision and a particular course of action (e.g., an eye movement), movement-selective neurons in the parietal and prefrontal cortices ramp up activity during the decision process, and this activity reaches a stereotypical peak just prior to movement onset when the neurons have reached a critical firing rate. This suggests that these neurons accumulate evidence for a perceptual decision (Shadlen \& Newsome 1996). Neuronal activity in these structures is also influenced by the prior probabilities of different stimuli and the reward structure of the task (Platt \& Glimcher 1999), just as is required for making optimal decisions (Gold \& Shadlen 2001). The activity of sensory neurons, however, does not always show a ramping profile and is often only weakly predictive of the ultimate decision (Britten et al. 1996, Shadlen et al. 1996). Some studies, therefore, have suggested that the role of sensory areas is restricted to providing momentary evidence (Law \& Gold 2008), which accumulates downstream into a decision. Yet other studies have revealed clear

signatures of the decision outcome in the early visual cortex. For example, when monkeys perform a curve-tracing task, the activity of the V1 neurons that represent the traced curve is enhanced, even if the animals mistakenly trace the wrong curve (Roelfsema \& Spekreijse 2001). The influence of a perceptual decision on the activity of sensory neurons is called choice probability, and this effect is presumably related to top-down influences on the visual cortex from areas that make the decision. Similarly, the reward structure of a curve-tracing task has been shown to influence V1 activity: Curves associated with larger rewards elicit stronger activity than curves associated with smaller rewards (Stănişor et al. 2013).

Although the choice probabilities in the curve-tracing task reflect the covert selection of one of a number of curves, Nienborg \& Cumming (2009) used choice probabilities to demonstrate a clear relation between neuronal activity in the early visual cortex and the perceptual representation of the sensory features of a stimulus. Monkeys were engaged in a binocular disparity discrimination task in which the information about the depth of a stimulus was presented in a time-varying and stochastic manner. The authors found that early disparity information influenced the monkey's decision and that later information was less effective, as if the animal had already made up its mind. Nevertheless, the activity of V2 neurons was related to the monkey's 
a
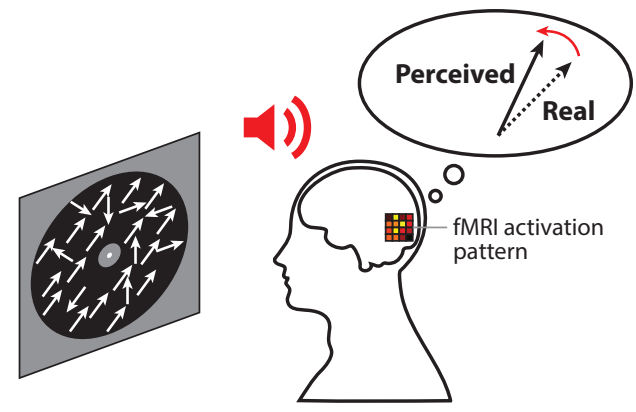

b

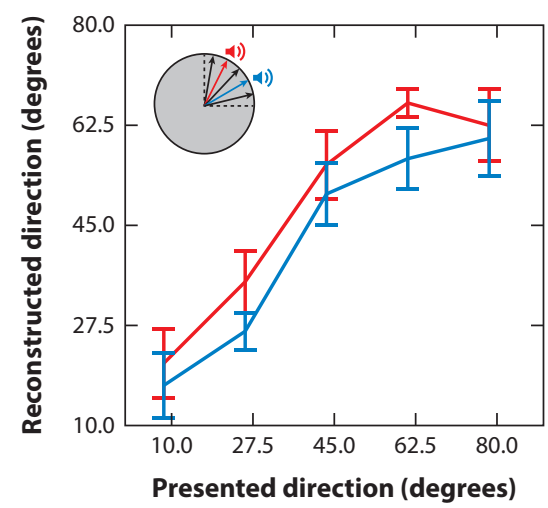

C

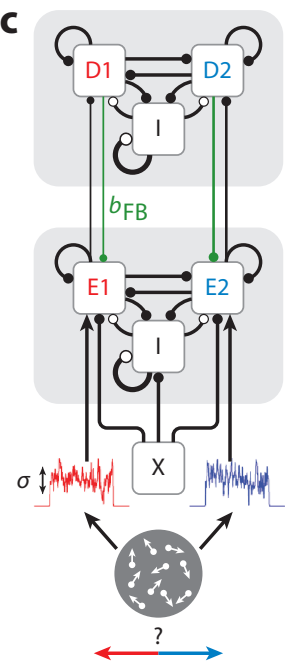

Figure 4

Influence of decision-making on activity in the early visual cortex. (a) Participants engaged in a motion identification task, in which they had to indicate the predominant direction of motion of a cloud of moving dots. Perceptual decisions were biased by presenting subjects with a tone prior to each trial, which indicated the likely direction of motion. Behavior and activation patterns were collected using fMRI. (b) Tone cues biased the perceived direction of motion as well as the sensory representation of motion direction in the early visual cortex, as reconstructed from the patterns of activity (Kok et al. 2013). (c) Recurrent network model composed of a sensory circuit (E1 and E2) that is coupled to a decision population (D1 and D2). Such a network can mimic the backpropagation of decision signals to sensory populations (Wimmer et al. 2015). Abbreviation: fMRI, functional magnetic resonance imaging. Panel $c$ was adapted from Wimmer et al. (2015).

decision for the full duration of the sensory sampling epoch, which suggested that the neurons received a disparity-related, feature-selective feedback signal from neurons in higher areas coding for the decision about disparity (Cohen \& Newsome 2008).

Functional magnetic resonance imaging studies in humans have also revealed an influence of sensory decisions on activity in the early visual cortex. For example, when predictive cues or stimulus statistics bias a perceptual decision, this alters choice-related signals in early visual cortical areas (Kok et al. 2012, 2014). Furthermore, manipulating participants' expectation about the likely direction of motion biases the perceptual decision as well as the neural representations in the early visual cortex (Kok et al. 2013) that can be gleaned from the BOLD activity patterns (Figure $4 \boldsymbol{a}, \boldsymbol{b}$ ). Together, these findings cast decision-making as a process of probabilistic inference, in which sensory and decision-related neurons are in a hierarchical and interactive relationship (Haefner et al. 2015, Nienborg \& Roelfsema 2015, Summerfield \& de Lange 2014). Neurocomputational modeling efforts (Wimmer et al. 2015; Figure 4c) have successfully described how interactions between decision and sensory neurons can give rise to the observed time courses of choice probability and evidence integration. As argued earlier, the role of the visual system may be likened to that of a dynamic blackboard, which not only provides information to a decision process but on which the unfolding decision process also writes.

\section{COMPLEX DECISIONS AND VISUAL ROUTINES}

Although many studies have focused on the neuronal processes underlying simple decisions, the neuronal correlates of decision-making have also been studied in more complex decision tasks. Lorteije et al. (2015), for example, recently studied the neuronal mechanisms of a hierarchical 
decision-making task in areas V1 and V4 of monkeys. The animals had to find the optimal path in an explicit decision tree (Figure 5a). They first had to make a decision at choice point L1, next to the fixation point, where they had to choose the branch with the brightest initial segment. After this, the monkeys encountered a second choice point (L2 or L2', depending on their choice at L1) where they had to choose the brightest branch once more and then select the circle at the end of this branch as the target for an eye movement (Figure 5a). Importantly, the luminance information at the three choice points was presented in a stochastic, time-varying manner so that the monkeys had to integrate luminance information over time to make optimal decisions. One might have expected that the monkeys would first decide about the choice point at L1 and then consider the downstream L2 decision so that they would not have to evaluate the irrelevant choice point L2'. However, the data suggested a different strategy. V1 and V4 neurons initially integrated luminance information for all choice points in parallel, starting at a latency of approximately $115 \mathrm{~ms}$ after the appearance of the stimulus. During this phase, the curves connected to the brighter branches were labeled with enhanced neuronal activity (Figure 5a). This was followed by a later phase during which the monkey's decision about all choice points became apparent, approximately $225 \mathrm{~ms}$ after stimulus onset (Figure 5a). At that point, the monkey's commitment to the L1 choice boosted the selection of the L2 decision behind the brighter L1 branch while evidence integration at the now irrelevant L2' decision declined. As a result of interactions between these local decision signals, the full path between the fixation point and the correct target for an eye movement was eventually labeled with enhanced neuronal activity in V1 and V4. Thus, in a decision-making task, the blackboard appears to represent the accumulation of evidence. The high spatial resolution representations in the early visual cortex may be essential for selecting one of a number of branches if they are close to one another in the image.

Although the decision tree in Figure $\mathbf{5} \boldsymbol{a}$ involved multiple similar decisions about brightness, there are also many tasks that involve a number of subtasks that are more heterogeneous (Ullman 1984). As a real world example, consider the task of making a cup of tea, which involves many substeps: locating the teacup, locating the teabag, putting the teabag in the cup, etc. In these cases, it can be useful to store the outcome of a decision on the retinotopic blackboard so that the next operator can act upon it. A location or feature that is the end product of one cognitive operation is tagged by enhanced neural activity, which can then be read out as the starting point for a subsequent operation. Empirical evidence for such a role of the visual system in multistep

\section{Figure 5}

Complex decisions and visual routines. (a) Hierarchical decision-making task. The monkeys had to mentally trace curves and make decisions at the bifurcations L1, L2, and L2' by choosing the path with the brightest curve elements. The visually driven activity in V1 and V4 was followed by a phase (starting at approximately $115 \mathrm{~ms}$ after stimulus onset) during which evidence for the three decisions was integrated in parallel. In a later phase, the path toward the correct eye movement target was labeled with enhanced activity as the result of interactions between the three local decisions while the incorrect path was suppressed. (Right) Time course of the visual response (black), the parallel integration phase (red; extra activity elicited by all the target branches), and the overall strategy (yellow; extra activity at L2 relative to L2'). (b) Search-then-trace task. The monkey had to search for a marker with the same color as the fixation point (T-marker) and then trace the target curve connected to this marker and make an eye movement to a target disc at the end of this curve. (c) The response enhancement due to search (red;228 ms) preceded the response enhancement due to tracing (yellow; $287 \mathrm{~ms}$ ). (d) Pseudocode that illustrates the concept of parameter transfer. The subroutines require variables as input and produce output that is stored in other variables. For example, Search_Color uses the variable Col as input, and it then retrieves Loc1, which is the location on the retinotopic map where this color is found. In a computer program, the variables refer to memory locations that hold the relevant information. The variable Loc1, for example, has to be stored so that it can be transferred to the subsequent tracing operation that has to start at this location. In the cortex, this variable can be stored as a focus of enhanced activity in the early visual cortex (red circle in c). Panel $a$ adapted from Lorteije et al. (2015). Panels $b-d$ adapted from Moro et al. (2010). 
tasks has been provided in the realm of visual search-and-trace tasks (Moro et al. 2010, Roelfsema et al. 2003).

One example task is illustrated in Figure $\mathbf{5} \boldsymbol{b}$. The monkey had to search for a marker that was cued by the color of the fixation point and he then had to mentally trace a curve that started at this marker to locate a disc that was the target of an eye movement. Activity in V1 revealed the

a

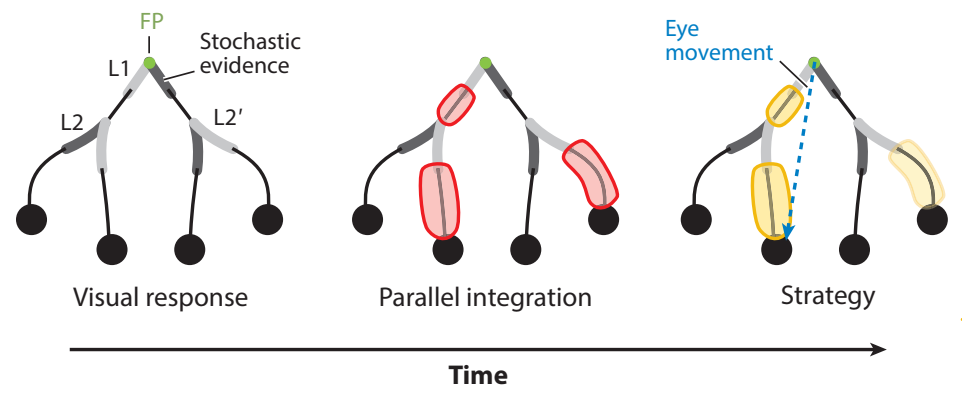

Hierarchical decision task

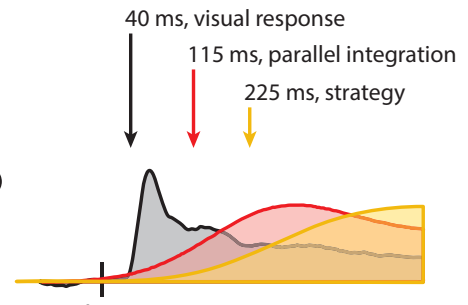

Stimulus onset

b

Search then trace

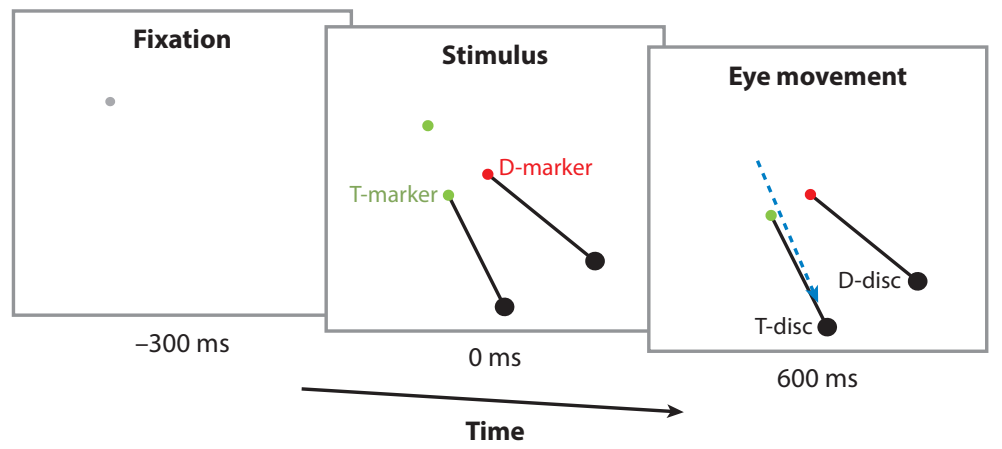

C

d

Visual Search Trace
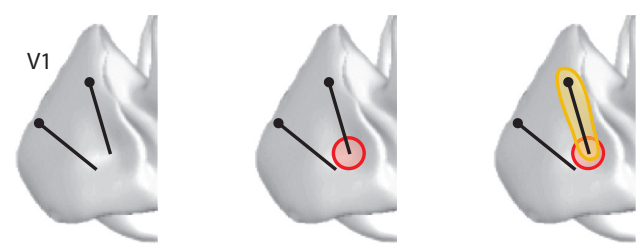

Search_then_Trace

\{

Col = Register_Color(FP)

Loc1 = Search_Color(Col)

Loc2 = Trace_Curve(Loc1)

Initiate_Saccade(Loc2)

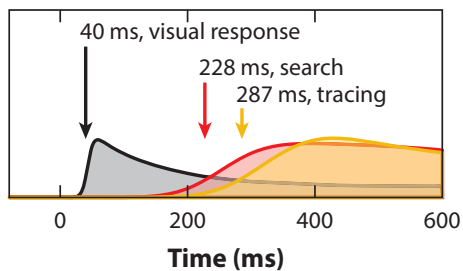

\} 
order of cognitive steps. First, neurons coded the visual attributes of the stimulus, at a latency of $40 \mathrm{~ms}$. This was followed by a phase during which the visual search process labeled the target marker with enhanced neuronal activity in V1 (after $228 \mathrm{~ms}$; Figure 5c). In the last phase, the V1 representation of the relevant curve, including the disc that was the target of the eye movement, was enhanced. Note how this task illustrates the utility of the early visual cortex as a cognitive blackboard, which enables the exchange of information between successive processing steps. It is likely that the color of the fixation point is first registered in regions of the visual and frontal cortices and that these areas feed back to earlier visual areas to highlight the location of the marker that has the same color (Hamker 2005, Spaak et al. 2016, van der Velde \& de Kamps 2001). This focus of enhanced activity in the retinotopic cortex can then be read out from the blackboard and used as the starting point for the subsequent tracing operation.

Figure $5 \boldsymbol{d}$ illustrates how a computer programmer would implement parameter transfer by storing the intermediate result of the search operator in a variable called Loc1, which is provided as a starting point for the subsequent tracing operator. Within a computer, these operations are associated with the copying of parameter values between memory locations in the computer during run time. The blackboard hypothesis proposes that the temporary storage can take place in the early visual cortex by highlighting the relevant features with enhanced neuronal activity, without the need to copy the values from one area to another. Area V1 may store spatial locations and elementary features in this way, whereas mid-level areas might store other features, such as the direction of motion (areas MT and MST) and color (area V4). These mid-level areas could also be the sites of write operations that highlight neuronal representations of task-relevant features so that subsequent read operations can use the response enhancement as input for successive cognitive steps.

\section{CHALLENGES FOR THE BLACKBOARD HYPOTHESIS}

The hypothesis that the early visual cortex can act as a cognitive blackboard faces a number of challenges. First, low-level attributes, such as luminance contrast, have a strong influence on the activity in the early visual cortex. Is it possible to highlight task-relevant features on the blackboard if they have a lower luminance contrast than irrelevant features? Previous work suggests that it is. Pooresmaeili et al. (2010) have demonstrated that it is possible to separately decode luminance contrast and behavioral relevance from V1 activity because there are neurons in the early visual cortex that are influenced by attention (A-neurons) and other neurons that are not $(\mathrm{N}-$ neurons). Higher visual areas can retrieve veridical information about luminance contrast from the $\mathrm{N}$-neurons, and they can also selectively read out what has been highlighted on the blackboard by evaluating the difference in activity between A- and N-neurons. Thus, variations in contrast across a visual scene need not interfere with blackboard functionality.

Eye movements, which occur on average three times per second, are another challenge for the blackboard. They shift the visual image across the retina and cause a new wave of feedforward input into the visual areas, which requires updating the pattern of response modulations on the blackboard. Interestingly, the approximately $300 \mathrm{~ms}$ fixation period between successive eye movements is longer than the processing time needed for the feedforward analysis of the visual scene. Therefore, the interval between eye movements provides a time window for information exchange via the blackboard. In this view, the length of the typical fixation reflects a trade-off between the need to exchange information via the blackboard and the advantage of acquiring new visual information by making a saccade. Moreover, eye movements do not completely erase the blackboard because higher areas may help to remap the blackboard's content after every saccade (Melcher \& Colby 2008). To test the impact of eye movements on V1 activity during curve tracing, 
Khayat et al. (2004) required monkeys to make eye movements across a curve-tracing stimulus. The monkeys previewed the stimulus so that they knew the course of the target curve before they made the saccade. If the eye movement brought a contour element into a V1 receptive field, the response enhancement occurred approximately $50 \mathrm{~ms}$ earlier if the stimulus had been previewed compared with when it had not been previewed. The implication is that task-relevant information highlighted on the blackboard is quickly restored after every saccade.

\section{CONCLUDING REMARKS}

In this review, we have put forward the view that cognitive operations may use the early visual cortex as a multiscale cognitive blackboard. This hypothesis is supported by a multitude of cognitive operations beyond the detection of the low-level features in which the visual cortex assists, some of which were reviewed here. Blackboard architectures have distinct computational advantages. They allow processors in higher visual areas to write their intermediate computational results to lower areas where they can be represented in a format that is accessible for other processors and can be easily read out for subsequent computations. The cognitive blackboard also enables analysis at multiple spatial scales (Hochstein \& Ahissar 2002). If the task can be solved at a coarse resolution, higher visual areas with large receptive fields can make quick progress, but when the task requires an analysis of fine details, lower areas come into play, usually at the cost of increased processing times. In previous work, we have suggested that sequences of read and write operations resemble the functionality of a Turing machine. In a Turing machine, the input and the content of memory jointly determine the next action, which then writes new information to the memory to bias the selection of subsequent actions (Zylberberg et al. 2011). Van der Velde \& de Kamps (2006) have proposed a related blackboard architecture for language processing that enables the flexible binding of words and concepts into larger sentences.

The evidence that higher cortical areas write to the blackboard of lower areas is compelling because many perceptual processes, such as texture segregation, visual search, and contour grouping, influence neuronal activity in the early visual cortex. These operations highlight visual features that are of current behavioral relevance. In addition, the blackboard may be used to exchange information when the stimulus is no longer in view, and we have reviewed evidence for the contributions of the early visual cortex to working memory and the mental manipulation of visual information (imagery). Determining the precise involvement of the early visual cortex in these mnemonic processes is an active topic of current research.

A crucial and complementary aspect of the blackboard functionality is that other brain areas read the information that has been stored in previous processing steps. We reviewed indirect evidence for read out in the texture-segregation task in which the pattern of response enhancement elicited by a figure predicts the precise landing position of the eye, which suggests that eye movement centers are sensitive to the pattern of activity on the blackboard. More direct tests of reading the blackboard rely on causal techniques to influence the response modulations in early visual areas without interfering with the initial propagation of information from lower to higher areas after the presentation of a new visual stimulus. Researchers have applied TMS pulses to disrupt activity in the early visual cortex at a point in time when the visual information has reached the higher visual areas. These TMS studies have revealed that interference with V1 activity indeed impairs accuracy in a number of cognitive processes that, according to our view, should rely on the blackboard, including texture segregation (Heinen et al. 2005), scene perception (Koivisto et al. 2011), visual search (Juan \& Walsh 2003), imagery (Kosslyn et al. 1999), and the storage of information in working memory (van de Ven et al. 2012). Future studies might use newly developed methods, such as optogenetics, to influence neuronal 
activity in a more precise manner and to further scrutinize the predictions of the blackboard hypothesis.

The cognitive blackboard provides a unifying framework for understanding the distinct, or even seemingly conflicting, hypotheses that the labeling of neurons with enhanced activity in the early visual cortex is the neuronal correlate of selective attention (Chelazzi et al. 2001), perceptual organization ( $\mathrm{Li}$ et al. 2006, Roelfsema 2006), decision-making (Lorteije et al. 2015, Nienborg \& Cumming 2009), visual routines (Moro et al. 2010), belief states (Nienborg \& Roelfsema 2015), or the value of visual stimuli (Baruni et al. 2015, Stănişor et al. 2013). These cognitive processes have often been studied separately, but they are all associated with the propagation of selection signals that cause task-relevant information to be highlighted on the blackboard. In decision-making tasks, the subject has to integrate sensory evidence to determine the next item that is relevant. Evidence integration is then associated with a gradual increase in the blackboard's representation of the item that needs to be selected. If the accumulation process goes awry and an erroneous item is highlighted, the subject makes an error, thus explaining the relation between neuronal activity in the early visual cortex and the subject's perceptual decision (Kok et al. 2013, Nienborg \& Roelfsema 2015). Furthermore, if items are associated with different reward levels, blackboard activity depends on the relative value of these items. Similarly, if the task demands the formation of perceptual groups, as is the case in texture-segregation and contour-grouping tasks, all features that belong to the task-relevant perceptual group are highlighted together on the blackboard (Roelfsema 2006, Roelfsema \& Houtkamp 2011). Psychologists describe this selection process as attentional selection. Thus, it is precisely the information that needs to be transferred from one cognitive step to the next in a visual routine that is within the focus of the subject's attention. We expect that future studies will continue to take advantage of the blackboard metaphor as a unifying framework for the many cognitive influences on neuronal activity in the early visual cortex, their computational advantages for information transfer in computational neuroscience, and the role of attention in cognitive psychology.

\section{SUMMARY POINTS}

1. The early visual cortex not only encodes sensory input but also assists in several cognitive operations, including attention, perceptual organization, working memory, imagery, and decision-making.

2. During these operations, the early visual system may serve as a cognitive blackboard, storing intermediate and final computations of other processors by highlighting relevant features with enhanced neuronal activity.

3. Higher areas can write to the blackboard by activating or enhancing the representation of features that are currently relevant and by suppressing features that are not. Intermediate computational results are thereby made available in a format that is easily read out for the computation of other processors.

4. If a visual object is selected for further processing, neurons in the early visual cortex that code the various features of the object enhance their response. As a result, these features are grouped in perception.

5. Neurons in the early visual cortex represent the stimuli held in working memory, and they also encode imagined stimuli during visual imagery. 
6. Feedforward and feedback connections subserve distinct computations and influence activity in different cortical layers.

7. The cognitive blackboard provides a unifying framework for understanding the seemingly distinct sets of results linking enhanced activity in the visual system to selective attention, perceptual organization, decision-making, or the value of stimuli. All these processes involve the propagation of selection signals, causing task-relevant information to be highlighted on the blackboard.

\section{FUTURE ISSUES}

1. Is the blackboard architecture unique to the early visual cortex or does this principle also apply to other perceptual and nonperceptual modules?

2. Is the modulation of neuronal activity in the early visual cortex causally relevant for these cognitive operations?

\section{DISCLOSURE STATEMENT}

The authors are not aware of any affiliations, memberships, funding, or financial holdings that might be perceived as affecting the objectivity of this review.

\section{ACKNOWLEDGMENTS}

We thank Chris Klink, Xing Chen, Jozsef Fiser, Peter Kok, Catherine Wacongne, and Jonathan Williford for feedback on an earlier version of the paper. P.R.R. was supported by grants from N.W.O. (The Netherlands Organisation for Scientific Research) (Vici grant 453-06-003, Brain and Cognition grant 433-09-208, and A.L.W. grant 823-02-010) and the European Union Seventh Framework Programme (“ABC,” PITN-GA-2008-290011, ERC Grant Agreement 339490, "Cortic_al_gorithms," and the Human Brain Project). F.P.de L. was supported by grants from N.W.O. (Vidi grant 452-13-016, Brain and Cognition grant 433-09-248, and MaGW grant 404-10-037), the James S. McDonnell Foundation (Understanding Human Cognition grant 220020373), and the European Union Horizon 2020 Programme (ERC Starting Grant 678286, "Contextvision").

\section{LITERATURE CITED}

Albers AM, Kok P, Toni I, Dijkerman HC, de Lange FP. 2013. Shared representations for working memory and mental imagery in early visual cortex. Curr. Biol. 23:1427-31

Albright TD. 2012. On the perception of probable things: neural substrates of associative memory, imagery, and perception. Neuron 74:227-45

Armstrong KM, Fitzgerald JK, Moore T. 2006. Changes in visual receptive fields with microstimulation of frontal cortex. Neuron 50:791-98

Baruni JK, Lau B, Salzman CD. 2015. Reward expectation differentially modulates attentional behavior and activity in visual area V4. Nat. Neurosci. 18:1656-63

Bhatt R, Carpenter GA, Grossberg S. 2007. Texture segregation by visual cortex: perceptual grouping, attention, and learning. Vis. Res. 47:3173-211 
Britten KH, Newsome WT, Shadlen MN, Celebrini S, Movshon JA. 1996. A relationship between behavioral choice and the visual responses of neurons in macaque MT. Vis. Neurosci. 13:87-100

Bullier J. 2001. Integrated model of visual processing. Brain Res. Brain Res. Rev. 36:96-107

Burt PJ, Adelson EH. 1983. The Laplacian pyramid as a compact image code. IEEE Trans. Commun. 31:532-40 Cavanagh P. 2011. Visual cognition. Vis. Res. 51:1538-51

Chelazzi L, Miller EK, Duncan J, Desimone R. 2001. Responses of neurons in macaque area V4 during memory-guided visual search. Cereb. Cortex 11:761-72

Chen M, Yan Y, Gong X, Gilbert CD, Liang H, Li W. 2014. Incremental integration of global contours through interplay between visual cortical areas. Neuron 82:682-94

Cohen MR, Newsome WT. 2008. Context-dependent changes in functional circuitry in visual area MT. Neuron 60(1):162-73

Duncan J, Humphreys GK, Ward R. 1997. Competitive brain activity in visual attention. Curr. Opin. Neurobiol. $7: 255-61$

Ekstrom LB, Roelfsema PR, Arsenault JT, Bonmassar G, Vanduffel W. 2008. Bottom-up dependent gating of frontal signals in early visual cortex. Science 321:414-17

Felleman DJ, Van Essen DC. 1991. Distributed hierarchical processing in the primate cerebral cortex. Cereb. Cortex 1:1-47

Ferrera VP, Rudolph KK, Maunsell JH. 1994. Responses of neurons in the parietal and temporal visual pathways during a motion task. F. Neurosci. 14:6171-86

Gilad A, Meirovithz E, Slovin H. 2013. Population responses to contour integration: early encoding of discrete elements and late perceptual grouping. Neuron 78:389-402

Gold JI, Shadlen MN. 2001. Neural computations that underlie decisions about sensory stimuli. Trends Cogn. Sci. 5:10-16

Gold JI, Shadlen MN. 2007. The neural basis of decision making. Annu. Rev. Neurosci. 30:535-74

Goldman-Rakic PS. 1995. Cellular basis of working memory. Neuron 14:477-85

Güçlü U, van Gerven MAJ. 2015. Deep neural networks reveal a gradient in the complexity of neural representations across the ventral stream. F. Neurosci. 35:10005-14

Haefner RM, Berkes P, Fiser J. 2015. The implications of perception as probabilistic inference for correlated neural variability during behavior. ArXiv:1409.0257 [q-bio.NC] http://arxiv.org/pdf/1409.0257.pdf

Hamker FH. 2005. The reentry hypothesis: the putative interaction of the frontal eye field, ventrolateral prefrontal cortex, and areas V4, IT for attention and eye movement. Cereb. Cortex 15:431-47

Harrison SA, Tong F. 2009. Decoding reveals the contents of visual working memory in early visual areas. Nature 485:632-35

Heinen K, Jolij J, Lamme VAF. 2005. Figure-ground segregation requires two distinct periods of activity in V1: a transcranial magnetic stimulation study. NeuroReport 16:1483-87

Hochstein S, Ahissar M. 2002. View from the top: hierarchies and reverse hierarchies in the visual system. Neuron 36:791-804

Houtkamp R, Roelfsema PR. 2010. Parallel and serial grouping of image elements in visual perception. F. Exp. Psychol. 36:1443-59

Houtkamp R, Spekreijse H, Roelfsema PR. 2003. A gradual spread of attention during mental curve tracing. Percept. Psychophys. 65:1136-44

Hung CP, Kreiman G, Poggio T, DiCarlo JJ. 2005. Fast readout of object identity from macaque inferior temporal cortex. Science 310:863-66

Itti L, Koch C. 2001. Computational modelling of visual attention. Nat. Rev. Neurosci. 2:194-203

Jolicoeur P, Ingleton M. 1991. Size invariance in curve tracing. Mem. Cogn. 19:21-36

Jolicoeur P, Ullman S, MacKay M. 1991. Visual curve tracing properties. 7. Exp. Psychol. 17:997-1022

Juan C-H, Walsh V. 2003. Feedback to V1: a reverse hierarchy in vision. Exp. Brain Res. 150:259-63

Kaas JH. 2008. The evolution of the complex sensory and motor systems of the human brain. Brain Res. Bull. 75:384-90

Khayat PS, Spekreijse H, Roelfsema PR. 2004. Correlates of transsaccadic integration in the primary visual cortex of the monkey. PNAS 101:12712-17

Koivisto M, Railo H, Revonsuo A, Vanni S, Salminen-Vaparanta N. 2011. Recurrent processing in V1/V2 contributes to categorization of natural scenes. F. Neurosci. 31:2488-92 
Kok P, Brouwer GJ, van Gerven MAJ, de Lange FP. 2013. Prior expectations bias sensory representations in visual cortex. F. Neurosci. 33:16275-84

Kok P, Failing MF, de Lange FP. 2014. Prior expectations evoke stimulus templates in the primary visual cortex. 7. Cogn. Neurosci. 26:1546-54

Kok P, Jehee JFM, de Lange FP. 2012. Less is more: Expectation sharpens representations in the primary visual cortex. Neuron 75:265-70

Kosslyn SM. 1996. Image and Brain. Cambridge, MA: MIT Press

Kosslyn SM, Ganis G, Thompson WL. 2001. Neural foundations of imagery. Nat. Rev. Neurosci. 2:635-42

Kosslyn SM, Pascual-Leone A, Felician O, Camposano S, Keenan JP, et al. 1999. The role of area 17 in visual imagery: convergent evidence from PET and rTMS. Science 284:167-70

Lamme VAF. 1995. The neurophysiology of figure-ground segregation in primary visual cortex. 7 . Neurosci. 15:1605-15

Law C-T, Gold JI. 2008. Neural correlates of perceptual learning in a sensory-motor, but not a sensory, cortical area. Nat. Neurosci. 11:505-13

LeCun Y, Bengio Y, Hinton G. 2015. Deep learning. Nature 521:436-44

Lee H, Simpson GV, Logothetis NK, Rainer G. 2005. Phase locking of single neuron activity to theta oscillations during working memory in monkey extrastriate visual cortex. Neuron 45:147-56

Lee TS, Yang CF, Romero RD, Mumford D. 2002. Neural activity in early visual cortex reflects behavioral experience and higher-order perceptual saliency. Nat. Neurosci. 5:589-97

Li W, Piëch V, Gilbert CD. 2006. Contour saliency in primary visual cortex. Neuron 50:951-62

Logothetis NK, Wandell BA. 2004. Interpreting the BOLD Signal. Annu. Rev. Physiol. 66:735-69

Long J, Shelhamer E, Darrell T. 2014. Fully convolutional networks for semantic segmentation. ArXiv: 1411.4038 [cs.CV]

Lorteije JAM, Zylberberg A, Ouellette BG, De Zeeuw CI, Sigman M, Roelfsema PR. 2015. The formation of hierarchical decisions in the visual cortex. Neuron 87:1344-56

Markov NT, Misery P, Falchier A, Lamy C, Vezoli J, et al. 2011. Weight consistency specifies regularities of macaque cortical networks. Cereb. Cortex 21:1254-72

Melcher D, Colby CL. 2008. Trans-saccadic perception. Trends Cogn. Sci. 12:466-73

Mendoza-Halliday D, Torres S, Martinez-Trujillo JC. 2014. Sharp emergence of feature-selective sustained activity along the dorsal visual pathway. Nat. Neurosci. 17:1255-62

Mesulam M. 2008. Representation, inference, and transcendent encoding in neurocognitive networks of the human brain. Ann. Neurol. 64:367-78

Moore T, Armstrong KM. 2003. Selective gating of visual signals by microstimulation of frontal cortex. Nature 421:370-73

Moro SI, Tolboom M, Khayat PS, Roelfsema PR. 2010. Neuronal activity in the visual cortex reveals the temporal order of cognitive operations. F. Neurosci. 30:16293-303

Muckli L, Petro LS. 2013. Network interactions: non-geniculate input to V1. Curr. Opin. Neurobiol. 23:195201

Mumford D. 1991. On the computational architecture of the neocortex. I. The role of the thalamo-cortical loop. Biol. Cybern. 65:135-45

Mumford D, Kosslyn SM, Hillger LA, Herrnstein RJ. 1987. Discriminating figure from ground: the role of edge detection and region growing. PNAS 84:7354-58

Nienborg H, Cumming BG. 2009. Decision-related activity in sensory neurons reflects more than a neuron's causal effect. Nature 459:89-92

Nienborg H, Roelfsema PR. 2015. Belief states as a framework to explain extra-retinal influences in visual cortex. Curr. Opin. Neurobiol. 32:45-52

Pasternak T, Greenlee MW. 2005. Working memory in primate sensory systems. Nat. Rev. Neurosci. 6:97-107

Pearson J, Naselaris T, Holmes EA, Kosslyn SM. 2015. Mental imagery: functional mechanisms and clinical applications. Trends Cogn. Sci. 19:590-602

Platt ML, Glimcher PW. 1999. Neural correlates of decision variables in parietal cortex. Nature 400:233-38

Pooresmaeili A, Poort J, Roelfsema PR. 2014. Simultaneous selection by object-based attention in visual and frontal cortex. PNAS 111:6467-72 
Pooresmaeili A, Poort J, Thiele A, Roelfsema PR. 2010. Separable codes for attention and luminance contrast in the primary visual cortex. F. Neurosci. 30:12701-11

Pooresmaeili A, Roelfsema PR. 2014. A growth-cone model for the spread of object-based attention during contour grouping. Curr. Biol. 24:2869-77

Poort J, Raudies F, Wannig A, Lamme VAF, Neumann H, Roelfsema PR. 2012. The role of attention in figure-ground segregation in areas V1 and V4 of the visual cortex. Neuron 75:143-56

Rainer G, Rao SC, Miller EK. 1999. Prospective coding for objects in primate prefrontal cortex. F. Neurosci. 19:5493-505

Rock I, Victor J. 1964. Vision and touch: an experimentally created conflict between the two senses. Science 143:594-96

Rockland KS, Virga A. 1989. Terminal arbors of individual "feedback" axons projecting from area V2 to V1 in the macaque monkey: a study using immunohistochochemistry of anterogradely transported Phaseolus vulgaris-leucoagglutinin. 7. Comp. Neurol. 285:54-72

Roelfsema PR. 2005. Elemental operations in vision. Trends Cogn. Sci. 9:226-33

Roelfsema PR. 2006. Cortical algorithms for perceptual grouping. Annu. Rev. Neurosci. 29:203-27

Roelfsema PR, Houtkamp R. 2011. Incremental grouping of image elements in vision. Atten. Percept. Psychophys. 73:2542-72

Roelfsema PR, Khayat PS, Spekreijse H. 2003. Subtask sequencing in the primary visual cortex. PNAS 100:5467-72

Roelfsema PR, Lamme VAF, Spekreijse H. 1998. Object-based attention in the primary visual cortex of the macaque monkey. Nature 395:376-81

Roelfsema PR, Lamme VAF, Spekreijse H, Bosch H. 2002. Figure-ground segregation in a recurrent network architecture. F. Cogn. Neurosci. 14:525-37

Roelfsema PR, Spekreijse H. 2001. The representation of erroneously perceived stimuli in the primary visual cortex. Neuron 31:853-63

Sakai K, Miyashita Y. 1991. Neural organization for the long-term memory of paired associates. Nature 354:152-55

Schall JD. 2001. Neural basis of deciding, choosing and acting. Nat. Rev. Neurosci. 2:33-42

Schlack A, Albright TD. 2007. Remembering visual motion: neural correlates of associative plasticity and motion recall in cortical area MT. Neuron 53:881-90

Self MW, Kooijmans RN, Supèr H, Lamme VAF, Roelfsema PR. 2012. Different glutamate receptors convey feedforward and recurrent processing in macaque V1. PNAS 109:11031-36

Self MW, van Kerkoerle T, Supèr H, Roelfsema PR. 2013. Distinct roles of the cortical layers of area V1 in figure-ground segregation. Curr. Biol. 23:2121-29

Serences JT, Ester EF, Vogel EK, Awh E. 2009. Stimulus-specific delay activity in human primary visual cortex. Psychol. Sci. 20:207-14

Shadlen MN, Britten KH, Newsome WT, Movshon JA. 1996. A computational analysis of the relationship between neuronal and behavioral responses to visual motion. F. Neurosci. 16:1486-1510

Shadlen MN, Newsome WT. 1996. Motion perception: seeing and deciding. PNAS 93:628-33

Shepard RN, Cooper LA. 1982. Mental Images and Their Transformations. Cambridge, MA: MIT Press

Shepard RN, Metzler J. 1971. Mental rotation of three-dimensional objects. Science 171:701-3

Spaak E, Fonken Y, Jensen O, de Lange FP. 2016. The neural mechanisms of prediction in visual search. Cereb. Cortex. In press. doi: 10.1093/cercor/bhv210

Stănişor L, van der Togt C, Pennartz CMA, Roelfsema PR. 2013. A unified selection signal for attention and reward in primary visual cortex. PNAS 110:9136-41

Stokes M, Thompson R, Cusack R, Duncan J. 2009. Top-down activation of shape-specific population codes in visual cortex during mental imagery. 7 . Neurosci. 29:1565-72

Summerfield C, de Lange FP. 2014. Expectation in perceptual decision making: neural and computational mechanisms. Nat. Rev. Neurosci. 15:745-56

Supèr H, Spekreijse H, Lamme VA. 2001. A neural correlate of working memory in the monkey primary visual cortex. Science 293:120-24

Thorpe S, Fize D, Marlot C. 1996. Speed of processing in the human visual system. Nature 381:520-22 
Ullman S. 1984. Visual routines. Cognition 18:97-159

van de Ven V, Jacobs C, Sack AT. 2012. Topographic contribution of early visual cortex to short-term memory consolidation: a transcranial magnetic stimulation study. F. Neurosci. 32:4-11

van der Velde F, de Kamps M. 2001. From knowing what to knowing where: modeling object-based attention with feedback disinhibition of activation. 7. Cogn. Neurosci. 13:479-91

van der Velde F, de Kamps M. 2006. Neural blackboard architectures of combinatorial structures in cognition. Behav. Brain Sci. 29:37-70

van Kerkoerle T, Self MW, Roelfsema PR. 2014. Effects of attention and working memory in the different layers of monkey primary visual cortex. Soc. Neurosci. Abstr. 263:13

Wannig A, Stanisor L, Roelfsema PR. 2011. Automatic spread of attentional response modulation along Gestalt criteria in primary visual cortex. Nat. Neurosci. 14:1243-44

Wimmer K, Compte A, Roxin A, Peixoto D, Renart A, de la Rocha J. 2015. Sensory integration dynamics in a hierarchical network explains choice probabilities in cortical area MT. Nat. Commun. 6:6177

Wolfson SS, Landy MS. 1998. Examining edge- and region-based texture analysis mechanisms. Vis. Res. 38:439-46

Yamins DLK, Hong H, Cadieu C, Solomon EA, Seibert D, DiCarlo JJ. 2014. Performance-optimized hierarchical models predict neural responses in higher visual cortex. PNAS 111:8619-24

Zaksas D, Pasternak T. 2006. Directional signals in the prefrontal cortex and in area MT during a working memory for visual motion task. 7. Neurosci. 26:11726-42

Zipser K, Lamme VAF, Schiller PH. 1996. Contextual modulation in primary visual cortex. F. Neurosci. 16:7376-89

Zylberberg A, Dehaene S, Roelfsema PR, Sigman M. 2011. The human Turing machine: a neural framework for mental programs. Trends Cogn. Sci. 15:293-300 
Annual Review of Vision Science

\section{Contents}

The Road to Certainty and Back

Gerald Westheimer

Experience-Dependent Structural Plasticity in the Visual System

Kalen P. Berry and Elly Nedivi ............................................. 17

Strabismus and the Oculomotor System: Insights from Macaque Models

Vallabh E. Das

Corollary Discharge and Oculomotor Proprioception: Cortical

Mechanisms for Spatially Accurate Vision

Linus D. Sun and Michael E. Goldberg

Mechanisms of Orientation Selectivity in the Primary Visual Cortex

Nicholas 7. Priebe

Perceptual Learning: Use-Dependent Cortical Plasticity

$W u L i$

Early Visual Cortex as a Multiscale Cognitive Blackboard

Pieter R. Roelfsema and Floris P. de Lange

Ocular Photoreception for Circadian Rhythm Entrainment in Mammals

Russell N. Van Gelder and Ethan D. Bubr

Probing Human Visual Deficits with Functional Magnetic Resonance Imaging

Stelios M. Smirnakis

Retinoids and Retinal Diseases

Philip D. Kiser and Krzysztof Palczewski

Understanding Glaucomatous Optic Neuropathy: The Synergy Between

Clinical Observation and Investigation

Harry A. Quigley

Vision and Aging

Cynthia Owsley

Electrical Stimulation of the Retina to Produce Artificial Vision

Fames D. Weiland, Steven T. Walston, and Mark S. Humayun 
Evolution of Concepts and Technologies in Ophthalmic Laser Therapy

Daniel Palanker

Low Vision and Plasticity: Implications for Rehabilitation

Gordon E. Legge and Susana T.L. Chung

The Human Brain in Depth: How We See in 3D

Andrew E. Welchman

Visual Object Recognition: Do We (Finally) Know More Now Than We

Did?

Isabel Gauthier and Michael 7. Tarr

3D Displays

Martin S. Banks, David M. Hoffman, Joobwan Kim, and Gordon Wetzstein

Capabilities and Limitations of Peripheral Vision

Ruth Rosenboltz

Visual Confidence

Pascal Mamassian

\section{Errata}

An online log of corrections to Annual Review of Vision Science articles may be found at http://www.annualreviews.org/errata/vision 